\title{
Appendix B:
}

\begin{tabular}{l|r}
\hline \multicolumn{1}{c|}{ Theme } & Frequency (\%) (n= 16) \\
\hline Watching them get sick/suffer & $7(43.75 \%)$ \\
\hline Not being able to visit/play & $5(31.25 \%)$ \\
\hline Not being able to help & $1(6.25 \%)$ \\
\hline I don't remember & $1(6.25 \%)$ \\
\hline Make me feel sad & $1(6.25 \%)$ \\
\hline Worried they might die & $2(12.50 \%)$ \\
\hline Not knowing how to treat them/respond & $1(6.25 \%)$ \\
\hline $\begin{array}{l}\text { B1: Michigan } 3^{\text {rd }}-5^{\text {th }} \text { grade students concerns pertaining to a friend with cancer: } 3^{\text {rd }}-5^{\text {th }} \text { grade respondents who indicated that they did have a friend } \\
\text { with cancer responded with these themes. }\end{array}$
\end{tabular}

\begin{tabular}{l|r}
\hline \multicolumn{1}{c|}{ Theme } & Frequency (\%) (n= 59) \\
\hline Watching them get sick/suffer & $20(33.90 \%)$ \\
\hline Not being able to visit/play & $14(23.73 \%)$ \\
\hline Not being able to help & $1(1.69 \%)$ \\
\hline Worried that they would die & $33(55.93 \%)$ \\
\hline Not knowing how to treat them/respond & $2(3.39 \%)$ \\
\hline Worried about stigma & $7(11.86 \%)$ \\
\hline Worried about contagious disease & $1(1.69 \%)$ \\
\hline Make me feel sad & $5(8.47 \%)$ \\
\hline Outlier & $2(3.39 \%)$ \\
\hline
\end{tabular}

B2: Michigan $3^{\text {rd }}-5^{\text {th }}$ grade students concerns pertaining to a friend with cancer: $3^{\text {rd }}-5^{\text {th }}$ grade respondents who indicated that they did not have a friend with cancer responded with these themes.

\begin{tabular}{l|r}
\hline \multicolumn{1}{c|}{ Theme } & Frequency (\%) (n = 19) \\
\hline Watching them get sick/suffer & $7(36.85 \%)$ \\
\hline Not being able to visit/play & $6(31.58 \%)$ \\
\hline Worried that they would die & $1(5.26 \%)$ \\
\hline Worried about stigma & $1(5.26 \%)$ \\
\hline Make me feel sad & $2(10.53 \%)$ \\
\hline Not knowing how to treat them/respond & $1(5.26 \%)$ \\
\hline Not being able to help & $2(10.53 \%)$ \\
\hline Outlier & $5(26.32 \%)$ \\
\hline $\begin{array}{l}\text { B3: } \text { Michigan } 6^{\text {rd }}-8^{\text {th }} \text { grade students concerns pertaining to a friend with cancer: } 6^{\text {rd }}-8^{\text {th }} \text { grade respondents who indicated that they did have a friend } \\
\text { with cancer responded with these themes. }\end{array}$
\end{tabular}

\begin{tabular}{l|r}
\hline \multicolumn{1}{c|}{ Theme } & Frequency (\%) (n= 42) \\
\hline Watching them get sick/suffer & $10(23.81 \%)$ \\
\hline Not being able to visit/play & $5(11.90 \%)$ \\
\hline Worried that they would die & $29(69.05 \%)$ \\
\hline Not knowing how to treat them/respond & $1(2.38 \%)$ \\
\hline Worried about stigma & $4(9.52 \%)$ \\
\hline Make me feel sad & $4(9.52 \%)$ \\
\hline Worried about how friends and family cope & $1(2.38 \%)$ \\
\hline Outlier & $.1(2.38 \%)$ \\
\hline $\begin{array}{l}\text { B4: Michigan } 6^{\text {rd }}-8^{\text {th }} \text { grade students concerns pertaining to a friend with cancer: } 6^{\text {rd }}-8^{\text {th }} \text { grade respondents who indicated that they did not have a } \\
\text { friend with cancer responded with these themes. }\end{array}$
\end{tabular}

\begin{tabular}{|c|c|c|c|}
\hline Theme & Grade $3^{\text {rd }}-5^{\text {th }}(n=16)$ & Grade 6-8 $(n=19)$ & P-Value \\
\hline Watching them get sick/suffer & $7(43.75 \%)$ & $7(36.84 \%)$ & 0.7391 \\
\hline Not being able to visit/play & $5(31.25 \%)$ & $6(31.58 \%)$ & 0.9833 \\
\hline Not being able to help & $1(6.25 \%)$ & $2(10.53 \%)$ & 0.6526 \\
\hline Make me feel sad & $1(6.25 \%)$ & $2(10.53 \%)$ & 0.6526 \\
\hline Worried they might die & $2(12.50 \%)$ & $1(5.26 \%)$ & 0.5820 \\
\hline Not knowing how to treat them/respond & $1(6.25 \%)$ & $1(5.26 \%)$ & 0.9003 \\
\hline \multicolumn{4}{|c|}{ B5: Comparison of concerns between $3^{\text {rd }}-5^{\text {th }}$ graders and $6^{\text {th }}-8^{\text {th }}$ graders who have a friend with cancer. } \\
\hline Theme & Grade $3^{\text {rd }}-5^{\text {th }}(n=59)$ & Grade 6-8 $(n=42)$ & P-Value \\
\hline Watching them get sick/suffer & $20(33.90 \%)$ & $10(23.81 \%)$ & 0.3772 \\
\hline Not being able to visit/play & $14(23.73 \%)$ & $5(11.90 \%)$ & 0.1965 \\
\hline Worried they might die & $33(55.93 \%)$ & $29(69.05 \%)$ & 0.2166 \\
\hline Not knowing how to treat them/respond & $2(3.39 \%)$ & $1(2.38 \%)$ & 0.7685 \\
\hline Worried about stigma & $7(11.86 \%)$ & $4(9.52 \%)$ & 0.7588 \\
\hline
\end{tabular}




\begin{tabular}{|c|c|c|c|}
\hline Make me feel sad & $5(8.47 \%)$ & $4(9.52 \%)$ & 0.8553 \\
\hline $\begin{array}{l}\text { B6: Comparison of concerns between } 3^{\text {rd }} \\
\text { cancer. }\end{array}$ & $5^{\text {th }}$ graders and $6^{\text {th }}-8^{\text {th }}$ grad & s who do not have a frier & with \\
\hline Theme & Grade $3^{\text {rd }}-5^{\text {th }}(n=75)$ & Grade $6^{\text {th }}-8^{\text {th }}(n=61)$ & P-Value \\
\hline Watching them get sick/suffer & $27(36.00 \%)$ & $17(27.87 \%)$ & 0.3595 \\
\hline Not being able to visit/play & $19(25.33 \%)$ & $11(18.03 \%)$ & 0.4061 \\
\hline Make me feel sad & $6(8.00 \%)$ & $6(9.84 \%)$ & 0.7672 \\
\hline Worried they might die & $35(46.67 \%)$ & $30(49.18 \%)$ & 0.8633 \\
\hline Not knowing how to treat them/respond & $3(4.00 \%)$ & $2(3.28 \%)$ & 0.8241 \\
\hline
\end{tabular}

